\title{
Adaptive Modulation and Coding scheme with intra- and inter-cell mobility for HSDPA system*
}

\author{
K. Ibrahimi ${ }^{\dagger}$, , R. El-Azouzi ${ }^{\dagger}$, S. K. Samanta ${ }^{\dagger}$ and E. H. Bouyakhf ${ }^{\ddagger}$ \\ ${ }^{\dagger}$ LIA/CERI, University of Avignon \\ 339 chemin des Meinajariès BP1228, 84911 cedex 9 - Avignon - France \\ Email: \{khalil.ibrahimi, rachid.elazouzi, sujit.samanta2\}@univ-avignon.fr \\ ${ }^{\ddagger}$ LIMIARF/FSR, Mohammed V-Agdal University \\ 4 Avenue Ibn Battouta B.P. 1014 Agdal - Rabat - Morocco \\ Email: bouyakhf@fsr.ac.ma
}

\begin{abstract}
The Adaptive Modulation and Coding (AMC) scheme which handles user's mobility issue plays a significant role to improve the desired quality of service in High Speed Downlink Packet Access (HSDPA) networks. We develop a resource allocation which maintains constant bit rate for real-time (RT) and non-real-time (NRT) calls independently of the user position in the cell. We present analytical results relating to user performance in terms of blocking and dropping probabilities, and the results relating to the system performance in term of total throughput. We propose a call admission control scheme to derive a good trad-off between the blocking and dropping probabilities, and the choice of scheduling that efficiently utilizes the available resources.
\end{abstract}

Index Terms-HSDPA, Call admission control, BCMP queueing, mobility, Erlang formula, fixed point.

\section{INTRODUCTION}

High-speed wireless network which has also been called 3.5G (Generation) for better data services is emerging as a promising solution to meet the increasing multimedia demands. The HSDPA of Release 5 concept introduces several new components to enhance downlink peak data rates, spectral efficiency, and Quality-of-Service (QoS) for packet services. These systems deliver high spectral efficiency by using TDMA-like strategy and opportunistic scheduling [1], [2]. The opportunistic standard scheduling adopted in HDR (high data rate) and HSDPA systems is the well-known Proportional Fair (PF) algorithm. In addition to opportunistic scheduling, two fundamental technologies those also rely on rapid adaptation of transmission parameters to the instantaneous radio conditions are used to increase the spectrum efficiency: Fast Link Adaptation technique and Fast Hybrid Automatic Repeat Request (HARQ) algorithm. The Release 5 and Release 6 of the 3GPP (3rd Generation Partnership Project) define both quadrature phase shift keying (QPSK) and 16 quadrature amplitude modulation (16-QAM) transmission in the downlink for HSDPA. The Release 7 introduces both 64-QAM for downlink connection HSDPA and 16-QAM for uplink connection HSUPA (High Speed Uplink Packet Access) [3]. High-speed downlink shared channel (HS-DSCH) is the transport channel, which is intended to carry high bit rates and

\footnotetext{
*This work was supported by a research contract with Maroc Telecom R\&D No. 10510005458.06 PI.
}

high load of packet traffic from the base station (Node-B) to the terminal. It is a new transport channel in HSDPA shared by a set of terminals in a time division manner per a shorter frame length of a transmission time interval (TTI) of $2 \mathrm{~ms}$ [4].

The use of higher order modulation and coding rate increases the bit rate of user, but requires more energy to maintain decoding performance at the receiver. Hence, the fast link adaptation is essential to extract any benefit by introducing higher order modulation and coding in the system. The standard link adaptation used in current wireless system is power control. However, HSDPA uses a constant transmission power during frame duration with fast link adaptation. Whereas the effective coding rate and modulation are adjusted according to radio condition. To avoid power rise as well as cell transmission power headroom requirements and to adapt the transmitted signal parameters to the continuously varying channel conditions, the link adaptation mechanisms must be included. One approach is to tightly coupled AMC and Scheduling. Link Adaptation to radio channel conditions is the baseline philosophy in HSDPA which serves users having favorable channel conditions. Users with bad channel conditions should wait for improved conditions to be served. HSDPA adapts the modulation and coding rates in parallel according to the instantaneous channel quality experienced by each user.

Li and Sampalli [5] developed an admission control scheme which handles only the intra cell mobility issue in the downlink of broadband wireless networks (HSDPA and WiMAX) with link adaptation. The authors decomposed the cell into a finite number of concentric circles regions. They adopted a simple propagation model where the path loss is a function of the distance between base station and mobile. They considered one class of service in the cell with link adaptation and modeled as a BCMP queueing chain network with fixed number of active users. The arrival rate of calls in each region depends only on the mobility (mean number of mobile users who move into the region) and not considered about new arrivals. The mean service time of a call in each region, depends only on the mobility (dwell time) not on call duration. This paper inspires us to develop a call admission control (CAC) in HSDPA cell that takes the intra cell and inter cell mobility 
scenarios and serves multiple classes of calls based on AMC scheme. We consider the arrival rate in closed form in each region as a function of mobility parameters (guard channel, area of geographical form, speed of mobility, average number of users) and the new arrival rate. The mean service time in each region is the minimum between the mobility (dwell time) and the mean duration of a call (mean size of a download file). We consider both classes of calls: real-time (RT) calls (e.g. streaming video, voice) and non-real-time (NRT) calls (e.g. FTP, email). The RT calls require constant bit rate value. Their service durations are independent of the consuming resources or bandwidth but depend on users' behavior. This is not the case for NRT applications. Service durations of NRT calls are dependent on mean amount (in bits) of download files.

In practice, the radio environment varies over time due to user mobility, shadowing and multi-path reflections. The cell is decomposed into concentric regions based on the AMC scheme. The intra cell mobility is modeled as the movement of users among the regions, where the users connected to the same base station with changing their modulation efficiency. Whereas the inter cell mobility users can change their base station to another base station. Each region is modeled by a queue with infinite servers as a BCMP queueing chain network. The users can move among these regions with migration/handoff rate as function of mobility parameters which are mentioned above. The notion of concentric regions has already been used by many other researchers [5], [6], [7], [8], [9]. Our CAC maintains for an active user of its class a constant bit rate anywhere in the cell area, in which the CAC adapts dynamically the needed resource for this user, when it moves from one region to another region. The needed resource becomes incapable to keep a same bit rate under the inter cell mobility and for this reason, the CAC algorithm increases this resource. Consequently, the number of calls in case of inter cell mobility which is less than the case of intra cell mobility is considered. Because the inter cell mobility decreases the cell capacity [10] in terms of accepted calls.

The main contributions in this paper are to develop a method for selecting an appropriate Modulation and Coding Scheme (MCS) according to the estimated channel condition, and to propose a new CAC mechanism which maintains a same QoS (constant bit rate) for all users of each class of calls (realtime (RT) and non-real-time (NRT)) anywhere in the area of the cell and gives priority to migrating or handoff calls by reserving the guard bandwidth than a new call in the intra cell and inter cell interferences. In addition, we derive a good trad-off between the blocking and dropping probabilities, and the choice of scheduling that efficiently utilizes the available resources.

The rest of the paper is as follows. In Section II, we describe the problem formulation. In Section III, we develop an analysis for this model with intra cell mobility based on Erlang formula. We propose a new CAC to enhance the performance for migrating calls. It is then extended to the multicell case in Section IV. Performance measures are contained in Section V. In Section VI, we give some numerical results and end with a concluding summary.

\section{Problem Formulation}

a) AMC scheme and cell decomposition: In wireless communication system, random fluctuations prevent the continuous use of highly bandwidth-efficient modulation, and therefore Adaptive Modulation and Coding (AMC) has become a standard approach in HSDPA system [11], [12]. As discussed in [13], the idea behind AMC is to dynamically change the modulation and coding scheme to the channel conditions. If good signal to interference-plus-noise ratio (SINR) is achieved, system can switch to the highest order modulation with highest code rates (e.g. 64-QAM with 3/4 Turbo Codes). If channel condition changes, system can shift to other low order modulation with low code rates (e.g. QPSK with $1 / 2$ Turbo Codes), but still not dropping call. The aim of selecting an appropriate Modulation and Coding Scheme (MCS) is to maintain an acceptable Bit Error Rate (BER) which should be lower than threshold, denoted by $B E R_{t h}$, for all users in the cell. Let $\left\{M_{1}, \ldots, M_{r}\right\}$ be a set of $r$ modulation and coding schemes. This set corresponds to the modulation and coding versus average channel instantaneous SINR. Hence, depending on the SINR, each user is assigned a best modulation and coding scheme such that $B E R \leq B E R_{t h}$. The average channel SINR values corresponding to the intersection points are chosen as the threshold values, denoted by $\left\{\gamma_{r}, \ldots, \gamma_{1}, \gamma_{0}=\infty\right\}$. These threshold points partitions the range of SINR into $r$ regions, denoted by $\left[\gamma_{i}, \gamma_{i-1}\right)$, for $i=1, \ldots, r$. The $k$ th modulation and coding scheme, namely $M_{k}$, is assigned to the region $\left[\gamma_{k}, \gamma_{k-1}\right)$ if the conditions $B E R\left(M_{k}, \gamma_{k}\right) \leq B E R_{t h}$ and $B E R\left(M_{k}, \gamma_{k+1}\right)>B E R_{t h}$ are satisfied (see Table I). Let we divide the cell into $r$ concentric circles of radii $R_{i}, i=1, \ldots, r$, and $S_{i}$ represents the corresponding surface of the $i$-th region. Each region corresponds to a specific modulation order as shown in Figure 1. So all users in a region have the same average radio condition and thus the same modulation and coding scheme.

\begin{tabular}{|c|c|c|c|}
\hline Received SINR (dB) & Radius & Modulation & Coding rate \\
\hline$\left[\gamma_{1}, \gamma_{0}\right)$ & $R_{1}$ & $64-$ QAM & $3 / 4$ \\
\hline$\left[\gamma_{2}, \gamma_{1}\right)$ & $R_{2}$ & 16-QAM & $3 / 4$ \\
\hline$\left[\gamma_{3}, \gamma_{2}\right)$ & $R_{3}$ & QPSK & $1 / 2$ \\
\hline$\left[\gamma_{4}, \gamma_{3}\right)$ & $R_{4}$ & BPSK & $1 / 2$ \\
\hline
\end{tabular}

Table I

MODULATIONS ORDER.

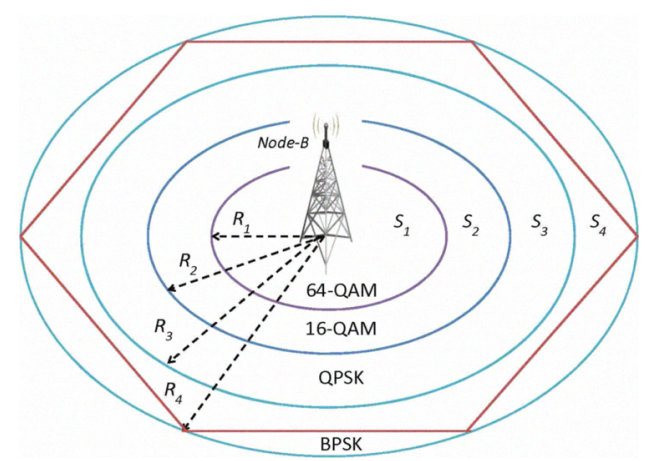

Figure 1. HSDPA Cell decomposed into concentric regions. 
b) Effective bandwidth per call: We assume that all HSDPA terminal users can access all $r$ modulations scheme. Let $P_{i, c}$ represent the HS-DSCH power transmitter from the Node-B to a mobile of class- $c$ in the region $i$. Let $q_{i}$ be the attenuation due to fading effects (path-loss, slow fading, fast fading, shadowing, multi-path, ...) between the base station and a mobile in the region $i$, see [6], [14]. Hence, the intra cell interference is given by

$$
I_{\text {intra }, i}=\alpha \frac{P_{t o t}-P_{i, c}}{q_{i}},
$$

where $P_{t o t}$ is the total HSDPA transmitter power served by Node-B and $\alpha$ is the downlink orthogonality factor [15]. Let $R_{c}$ denote the transmission bit rate of class- $c$ type call. All users of each class located in the region $i$ have the same average SINR value in $\left[\gamma_{i}, \gamma_{i-1}\right)$ and the same bit rate $R_{c}$ independently of their positions. The relationship among the bit rate, the instantaneous SINR in region $i$ of class- $c$ and the AMC scheme is given by

$$
\frac{R_{c}}{\left(\log _{2}(M) \tau\right)_{i}}=\frac{W}{S F_{16}} \frac{S I N R_{i, c}}{E_{c} / N_{0}},
$$

where $E_{c} / N_{0}$ is the required energy transmission per bit of class- $c$ type call to interference density, [6], [16]; $W$ is the chip rate; $N_{0}$ is the received noise floor; $\tau$ is the coding rate; $M$ is the modulation order; $S F_{16}$ is the HS-PDSCH (high speed-physical downlink shared channel) spreading factor of 16 and the $S I N R_{i, c}$ in region $i$ is given by

$$
S I N R_{i, c}=\frac{P_{i, c} / q_{i}}{I_{\text {intra }, i}+N_{0}}, \quad i=1, \ldots, r .
$$

The rest is to find the dynamic part of total bandwidth as function of region modulation order. This part is needed to guarantee for all users have same bit rate independently of their positions. Thus, from (1) and (3), we have

$$
\frac{S I N R_{i, c}}{1+\alpha S I N R_{i, c}}=\frac{P_{i, c}}{\alpha P_{t o t}+q_{i} N_{0}} .
$$

Let $M_{c}^{i}$ represent the number of HSDPA calls of class- $c$ in region $i$. Then the total transmitter power in the cell served by Node-B is expressed as

$$
P_{t o t}=P_{C C H}+P_{S C C H}+\sum_{i, c} M_{c}^{i} P_{i, c},
$$

where $P_{C C H}$ is the power of Common Channel and $P_{S C C H}$ is the power of Shared Control Channel. These powers are less than the total power $P_{t o t}$ and hence there exists a positive constant $\psi \in(0,1)$ such that $P_{C C H}+P_{S C C H}=\psi P_{t o t}$.

Now, let $\Lambda_{c}^{i}=\frac{P_{i, c}}{\alpha P_{t o t}+q_{i} N_{0}}$ (from (4)) and substitute the value of $P_{i, c}$ from it in (5), we get the total transmitter power of Node-B as

$$
P_{t o t}=\frac{N_{0} \sum_{i=1}^{r}\left(q_{i} M_{R T}^{i} \Lambda_{R T}^{i}+q_{i} M_{N R T}^{i} \Lambda_{N R T}^{i}\right)}{1-\psi-\alpha \sum_{i=1}^{r}\left(M_{R T}^{i} \Lambda_{R T}^{i}+M_{N R T}^{i} \Lambda_{N R T}^{i}\right)} .
$$

Define the effective bandwidth of user of class-c located in region $i$ as $\Delta_{c}^{i}=\alpha \Lambda_{c}^{i}$. Then, the needed bandwidth to maintain the same bit rate defined in (2) independently to user position is (using (4)) given by

$$
\Delta_{c}^{i}=\frac{R_{c}}{R_{c}+\frac{W}{S F_{16}} \frac{\left(\log _{2}(M) \tau\right)_{i}}{\alpha E_{c} / N_{0}}}, i=1, \ldots, r .
$$

We consider the system capacity or the total bandwidth capacity as $\Theta_{\epsilon}=1-\psi-\epsilon$, where $\epsilon>0$ is a very small positive number. Then, we have $\sum_{i=1}^{r}\left(M_{R T}^{i} \Delta_{R T}^{i}+M_{N R T}^{i} \Delta_{N R T}^{i}\right) \leq \Theta_{\epsilon}$. We note that this constraint ensures that no more than $\Theta_{\epsilon}$ can be occupied by all users of both classes (RT and NRT) simultaneously.

c) Modeling HSDPA cell: The call (RT and NRT) arrival processes are assumed to be independent Poisson process, and the call holding times are assumed to be independent exponentially distributed. Let $\lambda_{0, c}^{i}$ be the arrival rate of new calls of class- $c$ in region $i$. Let $1 / \mu_{R T}$ be the mean conversation time of an RT call in the system. We recall that the mean call duration of NRT calls in the system depends on the transmitting payload in bits, i.e., $\frac{1}{\mu_{N R T}}=\frac{E(P a y)}{R_{N R T}}$, where $E(P a y)$ is the mean file size [17]. Now, with $r$ regions in a cell, we have $2 r$ classes of calls in the system: $r$ classes of RT calls and $r$ classes of NRT ones. We consider each region behaves as a queue with infinite servers. Thus, the system (i.e., the cell) has $r$ queues and all the calls of a region remain in the same queue. If a call has the ability to leave its region toward an other one before to terminate, we talk about intra cell mobility scenario, whereas if a call of the system has not this ability, we talk about no intra cell mobility scenario which is developed in Section III.

\section{INTRA CELL MOBILITY MIGRATION}

d) No intra cell mobility: We consider here the incoming calls in region $i$ are due to new calls engaged by the mobiles belonging to the region $i$. In this case, each call accepted in the system remains in its region until the service termination. In this scenario, no region changes occur during the whole service time of a call. Thus, this situation corresponds to no intra cell mobility scenario. Let $n_{c}^{i}(t)$ be the number of calls of class- $c$ in progress at time $t$ in region $i$. The state of the system at time $t$ is defined by

$$
\vec{n}(t) \triangleq\left(n_{R T}^{1}(t), \ldots, n_{R T}^{r}(t), n_{N R T}^{1}(t), \ldots, n_{N R T}^{r}(t)\right) .
$$

Then, we model the process $\{\vec{n}(t), t>0\}$ as $2 r$-dimensions quasi-birth-and-death Markov chain, and has an unique stationary distribution $\pi(\vec{n})$, in the steady-state, with $\vec{n}=$ $\left(n_{R T}^{1}, \ldots, n_{R T}^{r}, n_{N R T}^{1}, \ldots, n_{N R T}^{r}\right)$. We note that a call of class- $c$ in region $i$ requires a part of total bandwidth capacity. Thus, we define the space of the admissible states as $A_{\Theta_{\epsilon}}=\left\{\vec{n} \in \mathbb{N}^{2 r} \mid \sum_{i=1}^{r}\left(n_{R T}^{i} \Delta_{R T}^{i}+n_{N R T}^{i} \Delta_{N R T}^{i}\right) \leq \Theta_{\epsilon}\right\}$. Then the probability to have a vector $\vec{n}$ of calls accepted in the system, i.e., $\vec{n} \in A_{\Theta_{\epsilon}}$ is given according to the BCMP theorem [18], [19] for multiple classes of calls as

$$
\pi(\vec{n})=\frac{1}{G\left(\Theta_{\epsilon}\right)} \prod_{i=1}^{r} \frac{\left(\rho_{R T}^{i}\right)^{n_{R T}^{i}}}{n_{R T}^{i} !} \frac{\left(\rho_{N R T}^{i}\right)^{n_{N R T}^{i}}}{n_{N R T}^{i} !}
$$


where $\rho_{c}^{i}=\lambda_{0, c}^{i} / \mu_{c}$ is the load of class-c calls in region $i$; $G\left(\Theta_{\epsilon}\right)$ is a normalizing constant computed using the condition $\sum_{\vec{n} \in A_{\Theta \epsilon}} \pi(\vec{n})=1$ as

$$
G\left(\Theta_{\epsilon}\right)=\sum_{\vec{n} \in A_{\Theta_{\epsilon}}} \prod_{i=1}^{r} \frac{\left(\rho_{R T}^{i}\right)^{n_{R T}^{i}}}{n_{R T}^{i} !} \frac{\left(\rho_{N R T}^{i}\right)^{n_{N R T}^{i}}}{n_{N R T}^{i} !} .
$$

A new call of class- $c$ will be blocked when it comes to the system and finds not enough available resources $\left(\Delta_{c}^{i}\right)$ for its service. Thus, the blocking probability of a new call of class- $c$ in region $i$ is given by

$$
B_{c}^{i}=1-\sum_{\vec{n} \in A_{\Theta_{\epsilon}-\Delta_{c}^{i}}} \pi(\vec{n})=1-\frac{G\left(\Theta_{\epsilon}-\Delta_{c}^{i}\right)}{G\left(\Theta_{\epsilon}\right)},
$$

where $G\left(\Theta_{\epsilon}-\Delta_{c}^{i}\right)$ is the normalizing constant calculated on space $A_{\Theta_{\epsilon}-\Delta_{c}^{i}}$.

e) With intra cell mobility : In the intra cell mobility scenario, the users connected to the same base station have the ability to change their regions (modulation order efficiency) by migration to another one. Then, the arrival rate of calls of class- $c$ in region $i$ is given by two cases. The first one, an user can make a call first time from region $i$ with rate $\lambda_{0, c^{i}}^{i}$ The second one, the call may come from region $j$ to region $i$ of the system due to migration possibilities with rate $\lambda_{c}^{j, i}$ $(i=1, \ldots, r, j \neq i)$. Note that $\lambda_{c}^{i, i}=\lambda_{0, c}^{i}$. A call of class$c$ which needs to migrate toward an other region has an high priority over a new call. In order to give the high priority to the migrating calls, we reserve eventually some bandwidth for the migrating/handoff calls (guard channel scheme [20]), denoted by $\Theta_{m}$. Furthermore, the total bandwidth or capacity becomes $\Theta_{\epsilon}=\Theta_{0}+\Theta_{m}$, where $\Theta_{0}$ is the normal bandwidth which can be used by the new arriving and migrating calls. If we consider $\delta$ such that $0 \leq \delta<1$, a portion of total bandwidth that the Operator can reserve to manage the mobility problem, then $\Theta_{m}=\delta \Theta_{\epsilon}$ and $\Theta_{0}=(1-\delta) \Theta_{\epsilon}$. As well, the reserved portion $\Theta_{m}$ of bandwidth depends on the manager policies (choice of $\delta$ ) used by an Operator to manage its network. The Operator can use $\Theta_{m}$ as a control parameter for determining the relative priority of migrating call over new call. Then the migrating call is blocked when all bandwidth $\Theta_{\epsilon}$ is used (busy), whereas the new call is blocked when the bandwidth $\Theta_{0}$ is occupied. The arrival rate of calls of class- $c$ in region $i$ is

$$
\lambda_{c}^{i}(\vec{n})= \begin{cases}\lambda_{0, c}^{i}+\sum_{j \neq i} \lambda_{c}^{j, i}, & \text { if } \vec{n} \in A_{0} ; \\ \sum_{j \neq i} \lambda_{c}^{j, i}, & \text { if } \vec{n} \in A_{\epsilon} \backslash A_{0},\end{cases}
$$

where

$$
\begin{aligned}
& A_{0}=\left\{\vec{n} \in \mathbb{N}^{2 r} \mid \sum_{i=1}^{r}\left(n_{R T}^{i} \triangle_{R T}^{i}+n_{N R T}^{i} \triangle_{N R T}^{i}\right) \leq \Theta_{0}\right\} \\
& A_{\epsilon}=\left\{\vec{n} \in \mathbb{N}^{2 r} \mid \sum_{i=1}^{r}\left(n_{R T}^{i} \triangle_{R T}^{i}+n_{N R T}^{i} \triangle_{N R T}^{i}\right) \leq \Theta_{\epsilon}\right\} .
\end{aligned}
$$

Let $1 / \mu_{i}^{m}$ be the mean dwell time of a mobile in region $i$. This means that the time for which a mobile is in the migration/handoff area depends on system parameters such as the speed, the direction of mobile travel and the region size. Then, the time spent by a call of class- $c$ in region $i$ is a random variable denoted by $T_{i}$ which is distributed, for simplicity, according to the exponential distribution with mean $1 / \mu_{i}^{m}$. Note that the variable $T_{i}$ depends on the mobility. Also the total completion duration of class- $c$ call in the system is a random variable denoted by $T_{c}$ which is distributed according to the exponential distribution with mean $1 / \mu_{c}$. Note that the variable $T_{c}$ depends on the amount of data can be sent. Then the activity time (holding time [21]) of class- $c$ call in region $i$ is a random variable denoted by $X_{c}^{i}=\min \left(T_{c}, T_{i}\right)$. The variables $T_{i}$ and $T_{c}$ are independent. Then the random variable $X_{c}^{i}$ is distributed according to the exponential distribution with rate $\mu_{c}+\mu_{i}^{m}$. This means that a call of class- $c$ occupies the resources in region $i$ for an exponentially distributed with mean duration $\frac{1}{\mu_{c}+\mu_{i}^{m}}$.

The state probability of the system in the steady-state is given by using the standard argument as for queueing networks. Hence, based on the BCMP theorem [19] for multiple classes with possible class changes, the state probability in the closed product-form is given with $\rho_{c}^{i}=\frac{\lambda_{c}^{i}(\vec{n})}{\mu_{c}+\mu_{i}^{m}}$ as follows:

$$
\pi(\vec{n})=\frac{1}{G_{\epsilon}} \prod_{i=1}^{r} \frac{\left(\rho_{R T}^{i}\right)^{n_{R T}^{i}}}{n_{R T}^{i} !} \frac{\left(\rho_{N R T}^{i}\right)^{n_{N R T}^{i}}}{n_{N R T}^{i} !}
$$

where $\vec{n} \in A_{\epsilon}$ and $G_{\epsilon}$ is the normalizing constant given by

$$
G_{\epsilon}=\sum_{\vec{n} \in A_{\epsilon}} \prod_{i=1}^{r} \frac{\left(\rho_{R T}^{i}\right)^{n_{R T}^{i}}}{n_{R T}^{i} !} \frac{\left(\rho_{N R T}^{i}\right)^{n_{N R T}^{i}}}{n_{N R T}^{i} !}
$$

The arrival rate $\lambda_{c}^{j, i}$ (as needed in (10)) is unknown and depends on the random number of users in the corresponding regions. These rates are calculated iteratively after assuming an initial value for them.

f) Calculation of migration arrival rates : The migration arrival rate of class- $c$ calls from region $j$ to region $i$ depends on the marginal expected number of calls in the region $j$. Let $p_{c, j}$ denote the probability that a call of class$c$ needs to migrate into other region from region $j$ before completing its service in the cell. Then we have the closed form expression of this probability as $p_{c, j}=P\left(T_{j}<T_{c}\right)=$ $\int_{0}^{\infty}\left(1-e^{-\mu_{j}^{m} t}\right) \mu_{c} e^{-\mu_{c} t} d t=\frac{\mu_{j}^{m}}{\mu_{j}^{m}+\mu_{c}}$. The rate at which the mobiles in the region $j$ try to migrate due to the mobility is $E\left[n_{c}^{j}(t)\right] p_{c, j} \mu_{j}^{m}$, where $E\left(n_{c}^{j}(t)\right)$ is the marginal expected mean number of class- $c$ calls in the region $j$ and given by

$$
E\left[n_{c}^{j}(t)\right]=\sum_{\vec{n} \in A_{\epsilon}} n_{c}^{j} \pi(\vec{n}) .
$$

Then, the arrival rate of migrating call in region $i$ from region $j$ is given by

$$
\lambda_{c}^{j, i}=\phi_{j, i} E\left[n_{c}^{j}(t)\right] p_{c, j} \mu_{j}^{m}, \quad i, j=1, \ldots, r ; j \neq i,
$$

where $\phi_{j, i}$ is the probability that an user in the region $j$ chooses the region $i$ for movement which depends on the mobility speed, the area geographical form of region $j$ and fading effects, such that $\sum_{j=1, j \neq i}^{r} \phi_{j, i}=1, i=1, \ldots, r$. In order to obtain the arrival rate $\left(\lambda_{c}^{i}(\vec{n})\right)$ of calls of class- $c$ in region $i$ from (10), we first need to compute the migration rate $\left(\lambda_{c}^{j, i}, j \neq i\right)$. To do this we follow the Algorithm 1: 


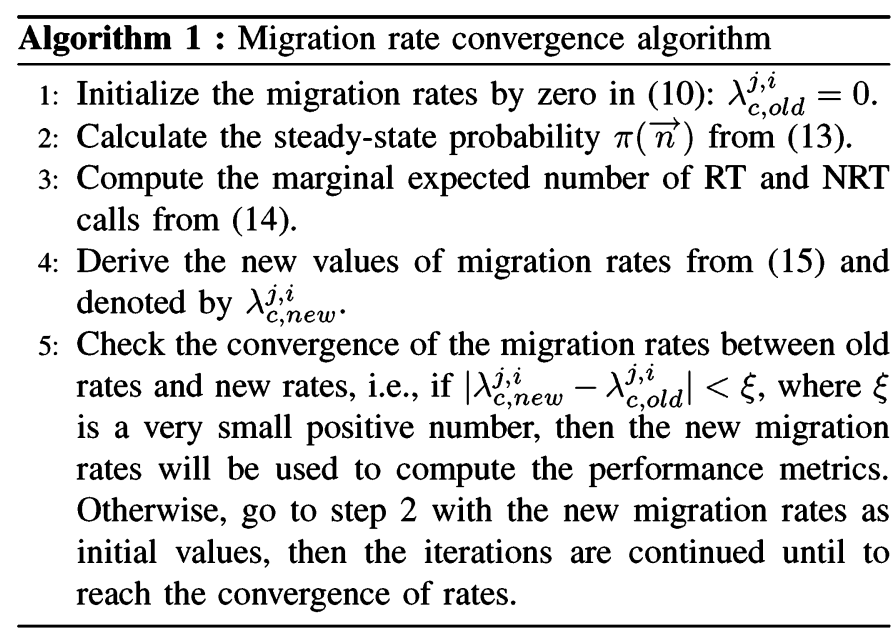

\section{INTER MULTI-CELLS NETWORK}

We consider here a multi-cells network composed of $l+1$ cells. Assume that all cells are identical and homogeneous. Here, the interference on an user in the region $i$ of the central cell 0 may be caused not only by the mobiles in its own cell, but also by the base stations of other cells (inter cells interference). To show the impact of the inter cell interferences on the effective bandwidth, we have

$$
I_{\text {inter }, i}=\sum_{j=1}^{l} \frac{P_{t o t, j}}{q_{i, j}}
$$

where $P_{t o t, j}$ is the total transmitter power served by base station of cell $j, q_{i, j}$ is the attenuation between an user located in the region $i$ of the central cell 0 and the interference cell $j$. Therefore, the relation (3) becomes

$$
S I N R_{i, c}=\frac{P_{i, c} / q_{i}}{I_{\text {intra }, i}+I_{\text {inter }, i}+N_{0}}, \quad i=1, \ldots, r .
$$

For simplicity, we consider in this homogeneous network, all cells have same total transmitter power, denoted by $P_{t o t}$. We use the $F$-factor at the central cell 0 defined in [22] as $F_{i, 0}=$ $q_{i} \sum_{j=1}^{l} \frac{1}{q_{i, j}}$ and for simplicity we replace $F_{i, 0}$ over all $i=$ $1, \ldots, r$ by its average value denoted by $F$. We recall that the capacity of wireless systems like CDMA/HSDPA is limited by the interference. In [10], the authors shown that the inter cell interference increases with the increase of interference $F$-factor. With the same arguments used to obtain (7), we deduce a new effective bandwidth including the inter cells interference to maintain a bit rate $R_{c}$ for an user independently of its position as

$$
\nabla_{c}^{i}=\frac{(\alpha+F) R_{c}}{\alpha R_{c}+\frac{W}{S F_{16}} \frac{\left(\log _{2}(M) \tau\right)_{i}}{E_{c} / N_{0}}}=(1+F / \alpha) \Delta_{c}^{i},
$$

where $\Delta_{c}^{i}$ is given by (7), for $i=1, \ldots, r$. So, we note that the new state spaces $A_{0}$ and $A_{\epsilon}$ will be computed by replacing $\Delta_{c}^{i}$ by the new bandwidth $\nabla_{c}^{i}$ in (11) and (12), respectively. The state of a cell of the network is $\vec{n}=$ $\left(n_{R T}^{1}, \ldots, n_{R T}^{r}, n_{N R T}^{1}, \ldots, n_{N R T}^{r}\right) \in \mathbb{N}^{2 r}$. We define the hand-off call when a call in the last region of the cell needs to migrate to other cells before terminating its service. Then the call arrival rate of class- $c$ to the last region $r$ in the central cell 0 , (as defined in (10)), including the hand-off arrival rate $\left(\lambda_{c}^{h o}\right)$ is

$$
\lambda_{c}^{r}(\vec{n})= \begin{cases}\lambda_{0, c}^{r}+\sum_{j=1}^{r-1} \lambda_{c}^{j, r}+\lambda_{c}^{h o}, & \text { if } \vec{n} \in A_{0} ; \\ \sum_{j=1}^{r-1} \lambda_{c}^{j, r}+\lambda_{c}^{h o}, & \text { if } \vec{n} \in A_{\epsilon} \backslash A_{0},\end{cases}
$$

where the migrating arrival rate $\lambda_{c}^{j, r}$ is computed by Algorithm 1 and the handover arrival rate $\lambda_{c}^{h o}$ is computed by Algorithm 2 (given in the sequel).

Since all cells are identical and thus, the marginal expected number of class- $c$ calls in the last region $r$ of each cell is

$$
E\left[n_{c}^{r}(t)\right]=\sum_{\vec{n} \in A_{\epsilon}} \pi(\vec{n}) n_{c}^{r} .
$$

As there are $l$ neighbor cells to the central cell and all are identical, therefore the hand-off arrival rate from all $l$ neighbor cells is

$$
\lambda_{c}^{h o}=l \beta_{l} E\left[n_{c}^{r}(t)\right] p_{c, r} \mu_{r}^{m},
$$

where $\beta_{l}$ is the probability that an user in the last region of any one of $l$ cells makes a handover to central cell.

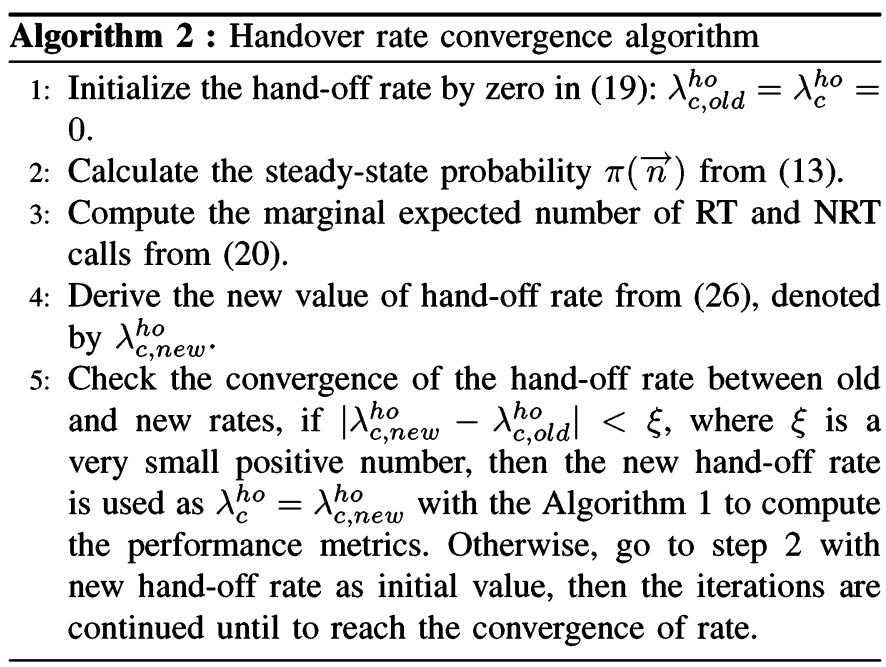

\section{PERFormanCE METRICS}

g) Blocking probability : If the system is in state $\vec{n} \in$ $E_{c}^{i}$ before a new arriving class- $c$ call in region $i$ then the call is blocked, where $E_{c}^{i}=\left\{\vec{n} \in A_{0} \mid \sum_{i=1}^{r}\left(n_{R T}^{i} \triangle_{R T}^{i}\right.\right.$ $\left.\left.+n_{N R T}^{i} \Delta_{N R T}^{i}\right)+\Delta_{c}^{i}>\Theta_{0}\right\}$. Thus, the blocking probability in region $i$ of class- $c$ call is given by

$$
P_{B}^{i, c}=\sum_{\vec{n} \in E_{c}^{i}} \pi(\vec{n}), i=1, \ldots, r .
$$

h) Dropping probability: In the literature, the term dropping probability refers to the blocking probability in handover [23] and it extended by other authors to intra cell mobility [24]. Here, we use the later case: a call of class- $c$ will be dropped when it moves to region $i$ and finds a lack of resources. So, dropping call depends largely on reserved bandwidth policies (choice of $\delta$ ) of the Operator. If a call migrates from region $j$ to region $i$, then this call requires the bandwidth $\Delta_{c}^{i}$ in region $i$. 
In order to maintain its communication without interruption, it needs to allocate the dynamic part of the bandwidth defined by: $\Delta_{c}^{i, j}=\Delta_{c}^{i}-\Delta_{c}^{j}$. Based on the availability of this bandwidth, we decide whether the call is dropped or not. We note that when $\Delta_{c}^{i, j} \leq 0$, the call is never dropped. But when $\Delta_{c}^{i, j}>0$, we check the available resources, i.e., if $\Delta_{c}^{i, j} \leq \Theta_{m}$ then the call is not dropped and if $\Delta_{c}^{i, j}>\Theta_{m}$ then the call will be dropped. Then the migrating class- $c$ call will be dropped if $\vec{n} \in A_{i, j}^{c}$, where $A_{i, j}^{c}=\left\{\vec{n} \in A_{\epsilon} \mid \sum_{i=1}^{r}\left(n_{R T}^{i} \triangle_{R T}^{i}\right.\right.$ $\left.\left.+n_{N R T}^{i} \Delta_{N R T}^{i}\right)+\Delta_{c}^{i, j}>\Theta_{\epsilon}\right\}$. Hence, the intra cell dropping probability (the ratio of the total migration or handover rate dropped in region $i$ to the total departure rate towards this region due to the mobility or call termination) in the region $i$ is

$$
P_{D}^{i, c}=\frac{\sum_{\vec{n} \in A_{i, i-1}^{c}} \mu_{i-1}^{m} n_{c}^{i-1} \pi(\vec{n})}{\sum_{\vec{n} \in A_{\epsilon}} n_{c}^{i-1}\left(\mu_{i-1}^{m}+\mu_{c}\right) \pi(\vec{n})}, i=2, \ldots, r .
$$

i) Average throughput and Bandwidth utilization: In the steady-state, we give the total throughput of the system in both cases: with intra cell (or inter cell) mobility having state $\vec{n} \in A_{\epsilon}$ and without intra cell mobility having state $\vec{n} \in A_{\Theta_{\epsilon}}$. They are given by

$$
T_{\text {cell }}^{\text {hsdpa }}=\sum_{\vec{n}} \pi(\vec{n}) \sum_{i=1}^{r}\left(n_{R T}^{i} R_{R T}+n_{N R T}^{i} R_{N R T}\right) .
$$

Another performance metric is the bandwidth utilization defined as the ratio of the effective bandwidth (the average value of the required bandwidths during calls) and the total bandwidth. It is given as

$$
U^{h s d p a}=\frac{\sum_{\vec{n}} \pi(\vec{n}) \sum_{i=1}^{r}\left(n_{R T}^{i} \triangle_{R T}^{i}+n_{N R T}^{i} \triangle_{N R T}^{i}\right)}{\Theta_{\epsilon}} .
$$

\section{NUMERICAL RESULTS}

We demonstrate numerical analysis for 3 concentric regions based on path-loss only as function of distance of user from his/her base station, for simplicity. This assumption is not essential and the theoretical results presented in this paper still hold in a more realistic radio environment. The HSDPA central cell is decomposed into three regions $(r=3)$ with AMC scheme: 64-QAM 3/4 (6 bits/symbol), 16-QAM 3/4 (4 bits/symbol) and QPSK 1/2 (2 bits/symbol). The require energy transmission per bit on thermal noise density $E_{c} / N_{0}$ is equal to $3.4 \mathrm{~dB}$ for $\mathrm{RT}$ calls, whereas $2.7 \mathrm{~dB}$ for NRT, the bit rate $R_{R T}$ is equal to $0.3 \mathrm{Mbps}$ and $R_{N R T}$ is 0.15 $M b p s$. The chip rate $W$ is equal to $3.84 \mathrm{Mbps}, \epsilon=10^{-5}$, the fraction of power for SCCH and $\mathrm{CCH}$ channels is $\psi=0.2$. The mean call duration for RT calls is equal to $120 \mathrm{sec}$, the download of files with mean size for NRT calls is equal to $E(P a y)=2$ Mbits and the orthogonality factor $\alpha$ is 0.2 . We study here low mobility case in the region with mean dwell time $300 \mathrm{sec}$. This corresponds to a pedestrian user moving at the speed of $5 \mathrm{Km} / \mathrm{h}$. The user in region $j$ moves only towards the neighboring region $(i=j \pm 1)$ with probability

$$
\phi_{j, i}= \begin{cases}1, & \text { if } j=1, i=2 \\ 1, & \text { if } j=r, i=r-1 \\ 1 / 2, & \text { if } 2 \leq j \leq r-1, i=j-1 \text { or } i=j+1 \\ 0, & \text { otherwise. }\end{cases}
$$

Then the arrival rate into region $i$ defined in (10) of class- $c$ call becomes

$$
\lambda_{c}^{i}(\vec{n})= \begin{cases}\lambda_{0, c}^{i}+\lambda_{c}^{i-1, i}+\lambda_{c}^{i+1, i}, & \text { if } \vec{n} \in A_{0} ; \\ \lambda_{c}^{i-1, i}+\lambda_{c}^{i+1, i}, & \text { if } \vec{n} \in A_{\epsilon} \backslash A_{0},\end{cases}
$$

where $\lambda_{c}^{0,1}=\lambda_{c}^{r+1, r}=0$ for $i=1, \ldots, r$. We assume that all $l$ neighbor cells of the central cell are identical and therefore, the probability $\beta_{l}$ is equal $1 / l$, and the hand-off arrival rate from all $l$ neighbor cells is

$$
\lambda_{c}^{h o}=l \frac{E\left[n_{c}^{r}(t)\right]}{l} p_{c, r} \mu_{r}^{m}=E\left[n_{c}^{r}(t)\right] p_{c, r} \mu_{r}^{m} .
$$

Impact without intra cell mobility: Figures 2 and 3 depict the blocking probabilities of new calls as function of new arrival RT call without mobility scenario in different regions (burst profile). The blocking probability in region 3 (QPSK) is higher as compare to region 1 (64-QAM) and region 2 (16QAM) as region 3 is far from the base station. The new call accepted in region 3 requires more bandwidth than a call in region 1, i.e., $\Delta_{R T}^{1}<\triangle_{R T}^{2}<\triangle_{R T}^{3}$ and $\Delta_{N R T}^{1}<\triangle_{N R T}^{2}<\triangle_{N R T}^{3}$. Moreover, an appreciable difference exists between blocking probabilities of classes of RT and NRT calls. This is due to the required bandwidth per class type, i.e., the class of RT call in our numerical environment requires more bandwidth than the class of NRT call. These results show that our CAC gives more priority for the calls who are near to the base station than far one. Furthermore, these probabilities increase with the increase of arrival rate. Figure 4 shows the effect of arrival rate of RT calls on average throughput without intra cell mobility scenario. Since all the calls accepted in the system with same bit rate, it is shown that the average throughput increases with the increase of new arrival rate of RT calls. We also see in this figure that the total bandwidth utilization instantaneously used by RT and NRT calls increases with the increase of arrival rate of RT calls.

Impact with intra cell mobility and F-factor: Figures 5 and 6 represent the blocking probabilities of new RT and NRT calls for different burst profile (efficiency modulation) as function of reserved portion $(\delta)$ of mobility problem. The blocking probabilities increase with the increase of $\delta$ as the CAC mechanism gives the priority to migrating call than the new call and the incoming region is away from the base station as well as the calls demand an high bandwidth. So the blocking probabilities mainly depend on the required bandwidth associated with the call modulation efficiency.

Figure 7 represents the dropping probabilities of RT and NRT calls with intra cell mobility scenario in logarithm scale. We observe that the dropping probability in region 2 (16QAM) for both classes RT and NRT is less than the dropping in region 3 (QPSK) and it decreases until the reserved bandwidth is less than $8 \%$. When the reserved bandwidth $\delta$ is more than $8 \%$, the dropping probability becomes constant for all traffics. This means that the migrating calls from region 1 to 2 as well as from region 2 to 3 are dropped constantly. We note that the migrating calls from region 2 to 1 (resp. from region 3 to 2) are never dropped by our CAC, because in region 1 (resp. region 2) they require less bandwidth than their occupied 


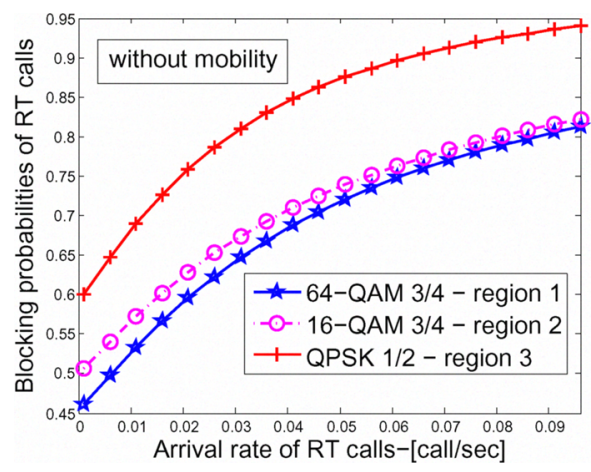

Figure 2. Blocking probabilities of RT calls versus new arrival rate of RT calls for $\lambda_{0, N R T}^{i}=10^{-5}$.

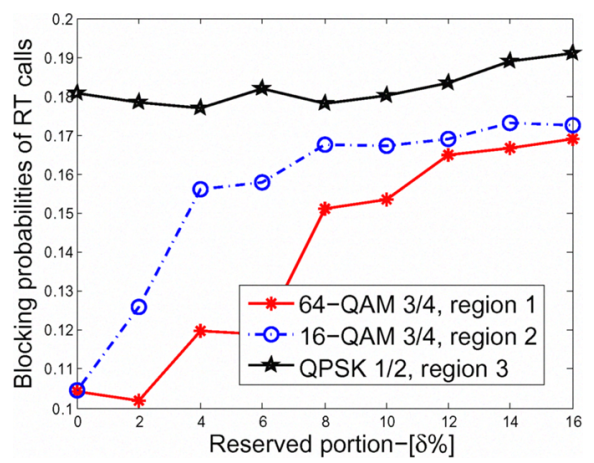

Figure 5. Blocking probabilities of RT calls versus the reserved portion $(\delta)$ for $\lambda_{0, R T}^{i}=2 * 10^{-3}$, $\lambda_{0, N R T}^{i}=10^{-3}$ and $1 / \mu_{i}^{m}=300 \mathrm{sec}$.

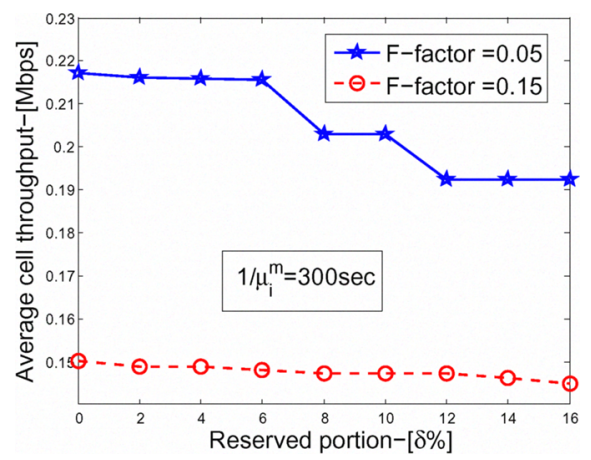

Figure 8. Average throughput versus the reserved portion $(\delta)$ of total bandwidth for $\lambda_{0, R T}^{i}=2 * 10^{-3}$, $\lambda_{0, N R T}^{i}=10^{-3}$ and $1 / \mu_{i}^{m}=300$ sec .

bandwidth in region 2 (resp. region 3). Figure 8 depicts the average throughput in terms of reserved portion of bandwidth with inter cell mobility scenarios. It is shown that the average throughput decreases with the increase of reserved bandwidth. The average throughput is influenced by high value of inter cell interference (high F-factor). From Figures 5 to 8, we can conclude that $\delta>8 \%$ does not provide good performances for blocking probability and average throughput, and the dropping probability of RT calls is acceptable in $0 \% \leq \delta \leq 8 \%$. Figure 9 represents the dropping probabilities of RT and NRT calls as function of reserved bandwidth $\delta$ with inter cell interference (F-factor). These results show that when $F$-factor increases, the HSDPA system capacity decreases (average throughput decreases) and thus the space of admissible states

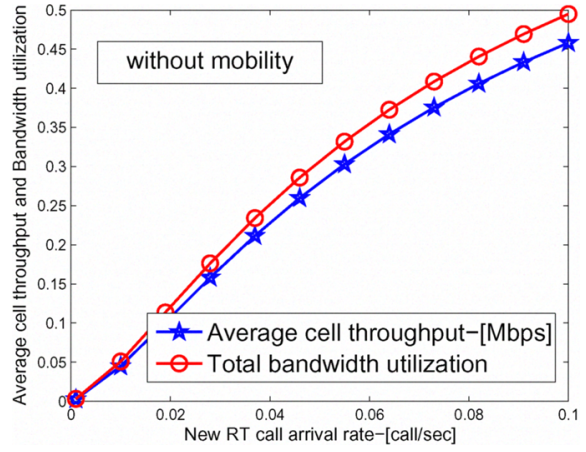

Figure 4. Average throughput and total bandwidth utilization versus new arrival rate of RT calls for $\lambda_{\mathrm{n} . \mathrm{NRT}}^{i}=10^{-5}$.

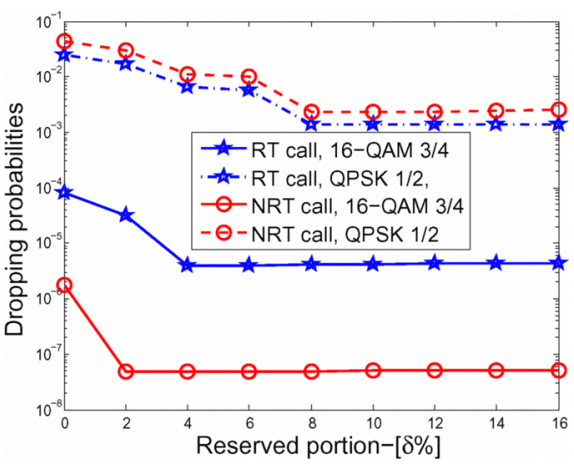

Figure 7. Dropping probabilities of calls versus the reserved portion $(\delta)$ for $\lambda_{0, R T}^{i}=2 * 10^{-3}$, $\lambda_{0, N R T}^{i}=10^{-3}$ and $1 / \mu_{i}^{m}=300 \mathrm{sec}$.

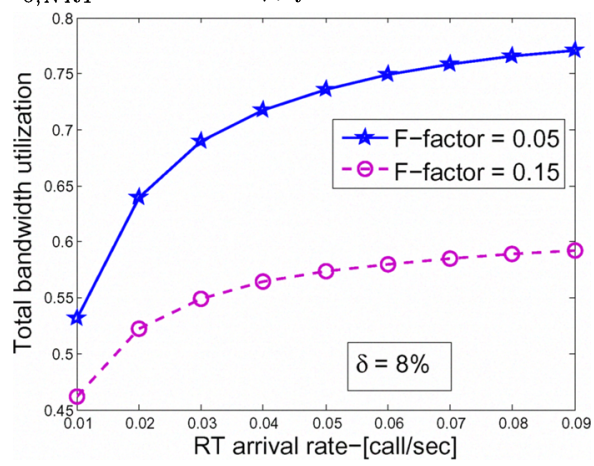

Figure 10. Total bandwidth utilization versus new arrival rate of RT calls for $\lambda_{0, N R T}^{i}=10^{-3}$ and $1 / \mu_{i}^{m}=300$ sec.

is reduced. These degradation of capacity observed in our results confirmed the result obtained in [10]. Furthermore, when $\delta$ is in $0 \% \leq \delta \leq 8 \%$, the dropping probabilities are high. Whereas for $\delta>8 \%$ the performance is good for both traffics RT and NRT. As a consequence, $0 \% \leq \delta \leq 8 \%$ is a good trade-off for both strategies: accept new call and migrating call with low dropping and blocking probabilities as well as acceptable average throughput. According to these results, the optimal value of guard-bandwidth $\Theta_{m}$ (choice of $\delta$ ) depends on the traffic pattern (migrating or handoff rate and new arriving call rate). Figure 10 shows the total bandwidth utilization occupancy of RT and NRT calls of the cell with inter cell mobility when the choice of Operator for mobility is $\delta=8 \%$. These results are plotted as function of new RT 
call arriving rate for different values of F-factor. We observe in this figure that $55 \%$ of total bandwidth is used for high value of $F$-factor $=0.15$ when $\mathrm{RT}$ arrival rate is equal to 0.04 $\mathrm{call} / \mathrm{sec}$, and also $73 \%$ of total bandwidth is used when $F$ factor $=0.05$. Figure 11 compares the dropping probabilities for RT and NRT calls when $\delta=8 \%$. The results are plotted in terms of new RT call arrival rate. We observe that our CAC has ability to keep the dropping probability relatively low for both regions 2 (16-QAM) and 3 (QPSK) even when $F$-factor is low. The CAC mechanism for RT call depends on the value of $F$-factor and the threshold of dropping calls.

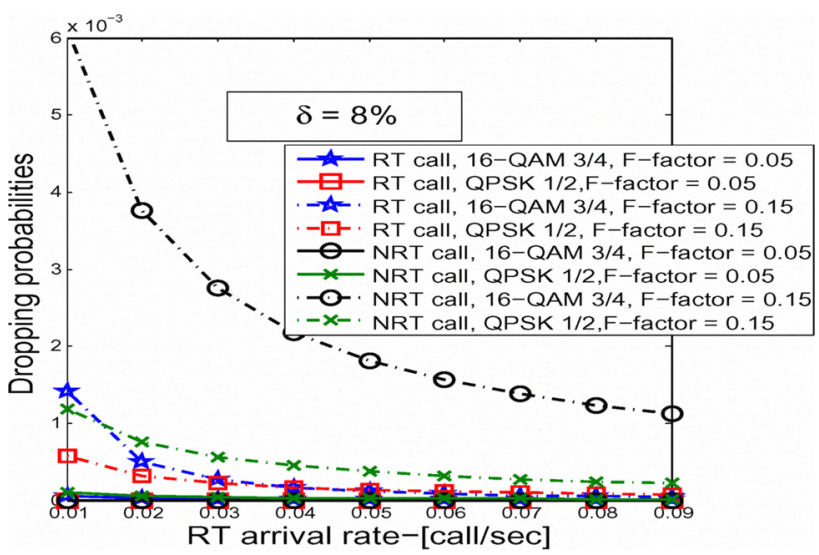

Figure 11. Dropping probabilities versus new arrival rate of RT calls for $\lambda_{0, N R T}^{i}=10^{-3}$ and $1 / \mu_{i}^{m}=300$ sec.

\section{CONCLUSION}

In this work, we focused on the UMTS extension HSDPA and presented an approach for serving users with a constant bit rate while changing between different modulation schemes, e.g., from 64-QAM to QPSK or vice versa. In HSDPA, the modulation is usually changed in dependence on the quality of the received signal, i.e., a good signal allows for using a modulation scheme with many bits per symbol and vice versa. Usually, a change from a higher to a lower modulation scheme is negatively experienced by the user, as it goes along with a decrease in the available bit rate. This problem is addressed by adapting the bandwidth when changing the modulation scheme with a goal to keep the initial bit rate. Our results show that the reserved bandwidth by Operator to manage its network depends largely on the traffic pattern (low blocking and low dropping). These results allow Operator to find a tradeoff as function of their customers need. The reserved bandwidth that belongs to this tradeoff gives increase largely the average throughput and low reject calls. We can study in future the HSDPA system considering several classes of user mobility. In fact the user mobility can be changed from very low mobility (user nomadic) to high mobility (user vehicular). These classes of mobility will be needed in order that the Operator provides a good QoS of different type of calls in both intra- and intercell mobility.

\section{REFERENCES}

[1] P. Bender, P. Black, M. Grob, R. Padovani, N. Sindhushyana, and S. Viterbi, "CDMA/HDR: A bandwidth-efficient high-speed wireless data service for nomadic users", IEEE Communications Magazine, Vol. 38(7), pp. $70-77,2000$

[2] R. Love, A. Ghosh, R. Nikides, L. Jalloul, and M. Cudak, "High speed downlink packet access performance", in Proceedings of IEEE 53rd Vehicular Technology Conference (VTC), Vol. 3, pp. 2234-2238, 2001.

[3] H. Holma, A. Toskala, K. Ranta-aho, and J. Pirskanen, "Nokia siemens networks high-speed packet access evolution in 3GPP Release 7", IEEE Communications Magazine, December 2007.

[4] H. Holma and A. Toskala, "HSDPA/HSUPA for UMTS : High Speed Radio Access for Mobile Communications", John Wiley \& Sons, Ltd. ISBN: 0-470-01884-4, 2006.

[5] J. Li and S. Sampalli, "Cell mobility based admission control for wireless networks with link adaptation", in Proceedings of ICC, 2007.

[6] T. Bonald and A. Proutière, "Wireless downlink data channels: user performance and cell dimensioning", in Proceedings of the 9th Annual International Conference on Mobile Computing and Networking, pp. 339352, New York, USA, 2003.

[7] S.E. Elayoubi, B. Elsaghir, and T. Chahed, "Other-cell interference in the downlink of multi-service UMTS", in Proceedings of IEEE 61st Vehicular Technology Conference (VTC), Vol. 1, pp. 354-358, 2005.

[8] A. Horrich, S.E. Elayoubi, and S.B. Jemaa, "On the impact of mobility and joint RRM policies on a cooperative WiMAX/HSDPA network", in Proceedings of WCNC 2008.

[9] S.E. Elayoubi, T. Chahed, and G. Hbuterne, "Mobility-aware admission control schemes in the downlink of third-generation wireless systems", IEEE Transactions on Vehicular Technology, Vol. 56(1), pp. 245-259, 2007.

[10] A.J. Viterbi, A.M. Viterbi, and E. Zehavi, "Other-cell interference in cellular power-controlled CDMA", IEEE Transactions on Communications, Vol. 42, pp. 1501-1504, 1994.

[11] R. Kwan, P.Chong, and M. Rinne, "Analysis of the adaptive modulation and coding algorithm with the multicode transmission", Journal Vehicular Technology Conference, Vol. 4, pp. 2007-2011, 2002.

[12] M. Nakamura, Y. Awad, and S. Vadgama, "Adaptive control of link adaptation for high speed downlink packet access (HSDPA) in W-CDMA", The 5th International Symposium on Wireless Personal Multimedia Communications, Vol. 2, pp. 382-386, 2002.

[13] R.C. Qiu, W. Zhu and Y. Zhang, "Third-generation and beyond (3.5G) wireless networks and its applications", IEEE International Symposium, Vol. 1 , pp. I-41-I-44, 2002.

[14] L. Sartori, S-E. Elayoubi ,B. Fourestie and Z. Nouir, "On the WiMAX and HSDPA coexistence", in Proceedings of IEEE ICC, pp. 5636-5641, 2007.

[15] N.B. Mehta, L.J. Greenstein, T.M. Willis, and Z. Kostic, "Analysis and results for the orthogonality factor in WCDMA downlinks", in Proceedings of IEEE Vehicular Technology Conference, pp. 100104, 2002.

[16] A.J. Viterbi, Cdma: Principles of spread spectrum communication, Addison- Wesley, 1995.

[17] A.B. Downey, "The structural cause of file size distributions", ACM SIGMETRICS Performance Evaluation Review, Vol. 29(1), pp. 328-329, 2001.

[18] F. Baskett, K.M. Chandy, R.R. Muntz, and F.G. Palaclos, "Open, closed and mixed networks of queues with different classes of customers", Journal of the ACM (JACM), Vol. 22(2), pp. 248-260, 1975.

[19] X. Chao, M. Miyazawa, M. Pinedo, and B. Atkinson, "Queuing networks: Customers, Signals and product form solutions", The Journal of the Operational Research Society, Vol. 52(5), pp. 600-601, 2001.

[20] D.Y. Hong and S.S. Rappaport, "Traffic model and performance analysis for cellular mobile radio telephone systems with prioritized and nonprioritized handoff procedures", IEEE Transactions on Vehicular Technology, Vol. 35(3), pp. 77-92, 1986.

[21] W.S. Jeon, and D.G. Jeong, "Call admission control for mobile multimedia communications with traffic asymmetry between uplink and downlink", IEEE Transactions on Vehicular Technology, Vol. 50(1), pp. 59-66, 2001

[22] K. Sipila, Z. Honkasalo, J. Laiho-Steffens, and A. Wackr, "Estimation of capacity and required transmission power of WCDMA downlink based on a downlink pole equation", in Proceedings of IEEE Vehicular Technology Conference, pp. 10021005, 2000.

[23] J.A.C. Bingham, "Multicarrier modulation for data transmission: An idea whose time has come", IEEE Communications Magazine, Vol. 28(5), pp. $5-14,1990$.

[24] S.E. Elayoubi and T. Chahed, "Admission control in the downlink of WCDMA/UMTS", Mobile and Wireless Systems, LNCS 3427, pp. 136$151,2005$. 